\title{
How to interpret LPV in roAp stars
}

\author{
H. Shibahashi ${ }^{1}$, D.W. Kurtz ${ }^{2}$, E. Kambe ${ }^{3}$ and D.O. Gough ${ }^{4}$ \\ ${ }^{1}$ Department of Astronomy, University of Tokyo, Tokyo 113-0033, Japan \\ email: shibahashi@astron.s.u-tokyo.ac.jp \\ ${ }^{2}$ Centre for Astrophysics, University of Central Lancashire, Preston PR1 2HE, UK \\ ${ }^{3}$ Department of Earth and Ocean Sciences, National Defense Academy, Yokosuka, \\ Kanagawa 239-8686, Japan \\ ${ }^{4}$ Institute of Astronomy, Madingley Road, Cambridge, CB30HA, UK
}

\begin{abstract}
Recent spectroscopic observations of roAp stars with high spectral resolution and high time resolution show line profile variations (LPV) which seem to be similar to those seen in rapidly rotating B-type line-profile variables. At first glance these line profile variations seem to be in disagreement with the mode identification based on the photometric observations. We propose an interpretation according to which the observed line profile variations are a manifestation of a shock wave in the high atmosphere near the magnetic polar regions, and show that the line profile variations can be still understood in the framework of the oscillation mode being consistent with the photometric observations.
\end{abstract}

Keywords. Stars: chemically peculiar, stars: oscillations, stars: variables: other, line: profiles

\section{Introduction: So what is the problem?}

It is almost a quarter of a century since rapid light variations in the magnetic Holmium star HD 101065 were discovered (Kurtz 1978). Similar rapid light variations with periods of $\sim 10 \mathrm{~min}$, being much shorter than the dynamical timescale of Main-Sequence stars, were discovered soon thereafter in several cool magnetic Ap stars (Kurtz 1982) and the concept of "rapidly oscillating Ap stars" was established. There are currently 33 known members of the class. Most observations of roAp stars have concentrated on the light variations. Until recently, very little work had been done on the effect of pulsation on the spectral lines. However, since the atmospheres of Ap stars are expected to be horizontally chemically inhomogeneous, it is expected that by using spectral lines of various elements one can get information on the spatial character of the eigenfunctions and, hopefully, identify the modes. High-resolution spectroscopic observations are required for this purpose. Recently some striking, new observational results have been reported (Savanov et al. 1999, Kochukhov \& Ryabchikova 2001a, 2001b, Balona \& Zima 2002, Kochukhov et al. 2002, Balona 2002, Balona \& Laney 2003, Kurtz, et al. 2003, 2004, Mkrtichian et al. 2003, Sachkov et al. 2004).

Kochukhov \& Ryabchikova (2001a) showed in the extremely slowly rotating $\left(P_{\text {rot }}>\right.$ $70 \mathrm{yr}$, see Leroy et al. 1994) roAp star, $\gamma$ Equ, spectacular line profile variations (LPV) in lines of Nd III and Pr III (see Fig. 1). The LPV in these lines of this star move only from blue-to-red. This blue-to-red shift pattern is observed in rapidly rotating $\mathrm{B}$ stars pulsating in intermediate $\ell$ sectoral modes. At a conference held in Mmabatho, South Africa in 2002 Luis Balona immediately called attention to this feature, and during a coffee break he came to one of us (HS), who gave an introductory review on roAp stars at this conference (Shibahashi 2003), to ask about any possible explanations for this phenomenon. Kochukhov \& Ryabchikova (2001a) interpreted the blue-to-red LPV of $\gamma$ Equ as being caused by oblique pulsation modes of $\ell=2$ or 3 and $m=-\ell$ or 


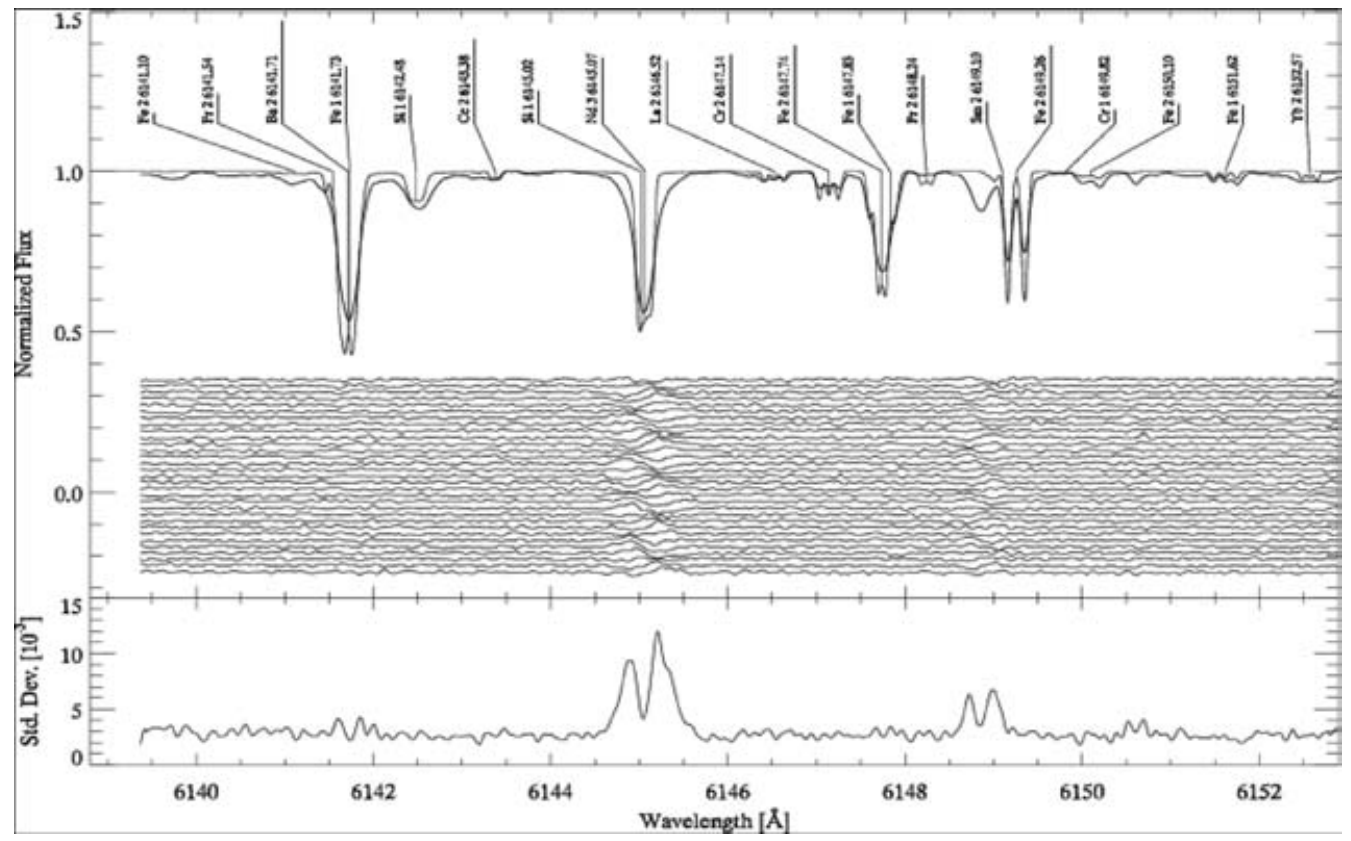

Figure 1. Spectral profile variation of $\gamma$ Equ taken by Kochukhov \& Ryabchikova (2001a) in the range of $\lambda \lambda 6140-6155$. The top part shows the time average of the spectra taken over the observational run and the theoretically synthesized spectra. The spectra were taken with $60 \mathrm{~s}$ exposure with a $40 \mathrm{~s}$ read-out interval. The middle part shows the difference of each spectra from the averaged spectrum. The time series are from the top to the bottom. The bottom part shows the standard deviation. Adopted from Kochukhov \& Ryabchikova (2001a).

$m=-\ell+1$. This result is inconsistent with the oblique pulsator model interpretation that the photometric variations are caused by low- $\ell$, axisymmetric modes. We wondered how a consistent explanation could be given or which mode identification was plausible, and, if we accepted the spectroscopic mode identification, why only $m=-\ell$ or $-\ell+1$ modes were observed. We got stuck for reasonable explanations, and started to think about the problem (Shibahashi et al. 2004).

Similar LPV are also seen in some other roAp stars in lines of Nd III and Pr III, which are believed to be formed in the upper atmospheric layer. However, some other photospheric lines do not show such monotonic blue-to-red shift.

\section{Case of the rapidly rotating $B$ stars}

When a star undergoes nonradial oscillations, different parts of the stellar surface move at different phases and this kind of motion produces a particular characteristic variation of line profile when combined with stellar rotation (see the Right panel of Fig. 2). The blue-to-red shift pattern is observed in rapidly rotating $B$ stars pulsating in intermediate $\ell$ sectoral modes $(\ell=m \sim 4-8)$. This is a manifestation of absorption bumps travelling across a rotationally broadened profile from blue to red. A typical example can be seen in a gray-scale plot for 19 Mon $\left(v \sin i=350 \mathrm{~km} \mathrm{~s}^{-1}\right)$ in Balona et al. (2002) (see the Left panel of Fig. 2).

However, $\gamma$ Equ has virtually no rotation $\left(P_{\text {rot }}>70 \mathrm{yr}\right.$ ) (Leroy et al. 1994), so this interpretation cannot be applicable to this star. 

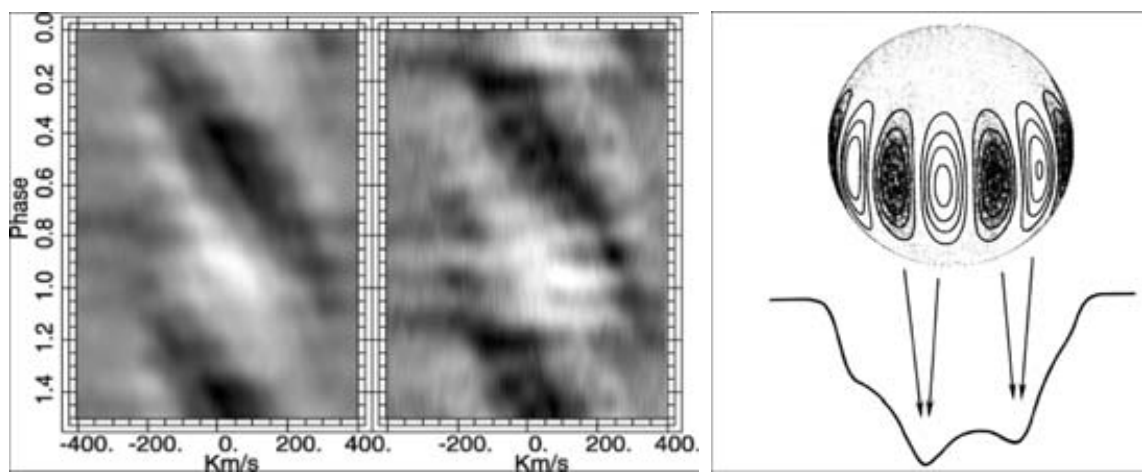

Figure 2. Left: LPV seen in the rapidly rotating B star, 19 Mon $\left(v \sin i=350 \mathrm{~km} \mathrm{~s}^{-1}\right)$. Adopted from Balona et al. (2002). Right: A schematic sketch of the explanation why the characteristic blue-to-red shift pattern in LPV are seen for a rapidly rotating B star oscillating in intermediate $\ell$ sectoral modes $(\ell=m \sim 4-8)$. Adopted from Vogt \& Penrod (1983).

\section{A new interpretation for LPV in roAp stars: shock wave?}

In the case of $\gamma \mathrm{Equ}$, there is no rotation to broaden the spectral lines and distinguish the blue approaching wing from the red receding wing. The LPV are expected to go back-and-forth, blue-to-red-to-blue, not just blue-to-red. To demonstrate this, we carried out theoretical simulations of LPV due to various modes for a nonrotating star. Some examples are shown in Fig. 3. As seen in this figure, the expected LPV due to the modes identified by Kochukhov \& Ryabchikova (2001a) are quite different from the observed LPV in Nd III and Pr III, hence their mode identification cannot be correct. The LPV due to other modes show essentially the same feature, the blue-to-red-to-blue variation. This is true for $\ell=1$ and $m=0$ modes, which are implied from the photometric observations.

$\mathrm{SPH} L=2 \quad M=-1 \quad \mathrm{AMP}=10 \mathrm{~km} / \mathrm{s} \quad C=10 \mathrm{~km} / \mathrm{s} \mid \mathrm{ncl}=130 \quad \mathrm{SPH} L=2 \quad M=-2 \mathrm{AMP}=10 \mathrm{~km} / \mathrm{s} \quad \mathrm{C}=10 \mathrm{~km} / \mathrm{s} \quad \mathrm{Incl}=130$
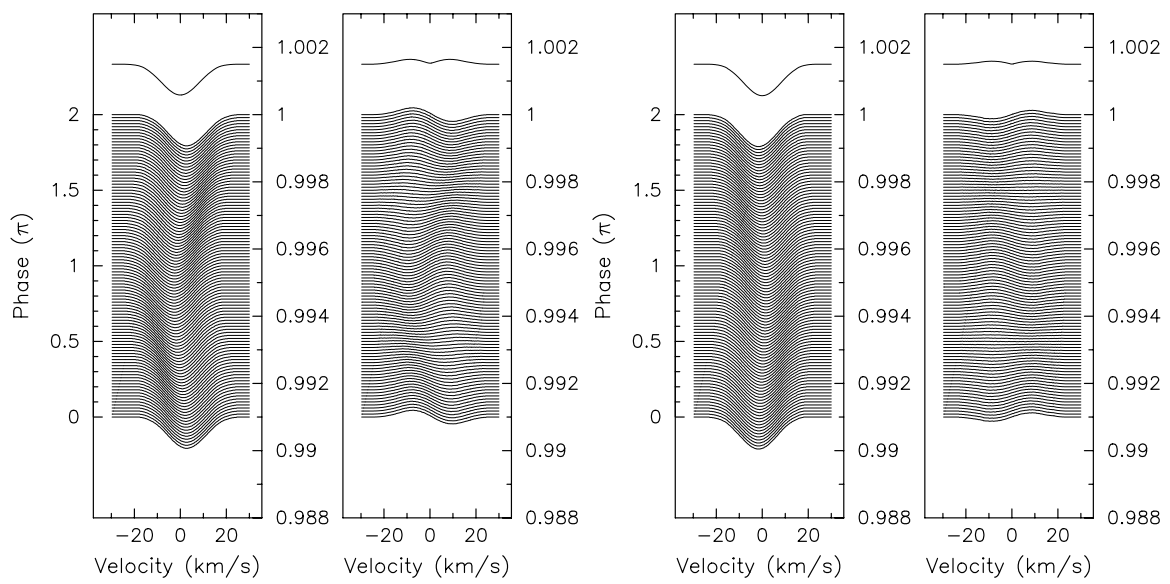

Figure 3. Theoretically expected LPV due to an $\ell=2, m=1$ mode (left), and an $\ell=2$, $m=2$ mode (right) for a nonrotating star. The pulsation amplitude is assumed to be $10 \mathrm{~km} \mathrm{~s}^{-1}$ and the line profile is assumed to have an intrinsic line width corresponding to a velocity of $10 \mathrm{~km} \mathrm{~s}^{-1}$. The aspect angle between the pulsation axis and the line-of-sight is assumed to be $130^{\circ}$. For each mode the LPV for one pulsation cycle are shown on the left panel, and the differences between the LPV and the average profile (shown top left) are displayed in the right panel. Clearly, the LPV move from blue to red and back again, not just from blue-to-red. The top of the right panel for each mode shows the standard deviation. 
Why do the LPV in Nd III and Pr III show a monotonic blue-to-red movement, while the photometric observations imply axisymmetric dipole oscillations, from which blue-tored-to-blue LPV are expected? To begin with, what is the cause of the line broadening in the nonrotating star? The presence of a strong magnetic field induces Zeeman splitting of the spectral lines. The resultant broadening, together with the thermal broadening, of Nd III $\lambda 6145$ is estimated to correspond to a velocity field of $\sim 9 \mathrm{~km} \mathrm{~s}^{-1}$ (Kochukhov 2004, private communication), which is smaller than the observed value, $\sim 18 \mathrm{~km} \mathrm{~s}^{-1}$. This means that the line-width itself is unexpectedly broad and that we need some explanation.

We propose that the observed line width is essentially due to the pulsation velocity. Accordingly the Nd III $\lambda 6145$ line-forming layer is moving with a maximum speed of $18 \mathrm{~km} \mathrm{~s}^{-1}$ in one pulsation cycle. This maximum speed is much higher than the radial velocity pulsation amplitudes so far detected from other spectral lines in other roAp stars, of the order several $\mathrm{km} \mathrm{s}^{-1}$, in exceptional cases, but usually much less. In the very high atmosphere, the oscillations might be evanescent. Even though the energy density, $\rho \boldsymbol{v}^{2}$, might decrease exponentially with height, the velocity amplitude $\boldsymbol{v}$ itself increases with height because the density $\rho$ decreases with height more rapidly than the energy density. Hence if Nd III $\lambda 6145$ forms in a high layer, the large amplitude is not unnatural.

The important point is that this maximum speed seems to be faster than the sound speed of the atmosphere. The pulsation amplitude in velocity is expected to increase with the decrease of the density with height. So the amplitude in velocity naturally exceeds the sound speed at a certain level, and a shock wave is generated. When this shock wave propagates through the layer under consideration, the layer is suddenly kicked upward by the shock wave and then falls down after reaching the peak height, at which the speed of the layer is zero. This process repeats and the resultant variation in the Doppler shift is qualitatively in agreement with the observed one in $\mathrm{Nd}$ line, blue-to-red, then sudden back to blue, and then blue-to-red again, which is apparently similar to the LPV seen in the rapidly rotating B stars (Shibahashi et al. 2004). This picture may be consistent with the mode identification from the photometric observations, which imply the axisymmetric dipole modes whose symmetry axis coincides with the magnetic axis, itself inclined to the rotation axis of the star (the oblique pulsator model). With this picture, we expect the following (Shibahashi et al. 2004):

- The LPV should be sinusoidal for the spectral lines formed in layers where the wave motion is subsonic.

- The LPV should be sinusoidal for chemical elements that are not concentrated in the magnetic polar regions, so their pulsation velocities remain subsonic.

- The LPV should show monotonic blue-to-red motion only for the lines formed in the high atmosphere near the magnetic polar regions.

We simulate LPV in the Nd III line of $\gamma$ Equ based on this hypothesis. As the most extreme case, we consider the case that the temporal variation is saw-toothed. The eigenfunction is given as $Y_{\ell}^{m}(\theta, \phi)$, but its temporal variation is assumed to be not sinusoidal but saw- toothed $-\sum_{n=1}^{10} n^{-1} \exp (i n \sigma t)$. Figure 4 shows the LPV based on this model for $\ell=0$ or $\ell=1, m=0$ or $\ell=1, m=1$ with a large inclination angle $i$, or $\ell=2$, $m=0$ with $i \simeq 0$, are in agreement with the observed LPV in lines of Nd III and Pr III. Note that the mode identification $\ell=1, m=0$ is consistent with that deduced from photometric observations (the oblique pulsator model). The oscillation mode is then still consistent with the photometric observations. 
$\mathrm{SPH} L=1 \quad \mathrm{M}=0 \quad \mathrm{AMP}=20 \mathrm{~km} / \mathrm{s} \quad \mathrm{C}=10 \mathrm{~km} / \mathrm{s} \quad \mathrm{ncl}=30 \mathrm{~S}-3 \mathrm{SPH} L=1 \quad \mathrm{M}=-1 \quad \mathrm{AMP}=20 \mathrm{~km} / \mathrm{s} \quad \mathrm{C}=10 \mathrm{~km} / \mathrm{s} \quad \mathrm{ncl}=90 \mathrm{~S}-3$
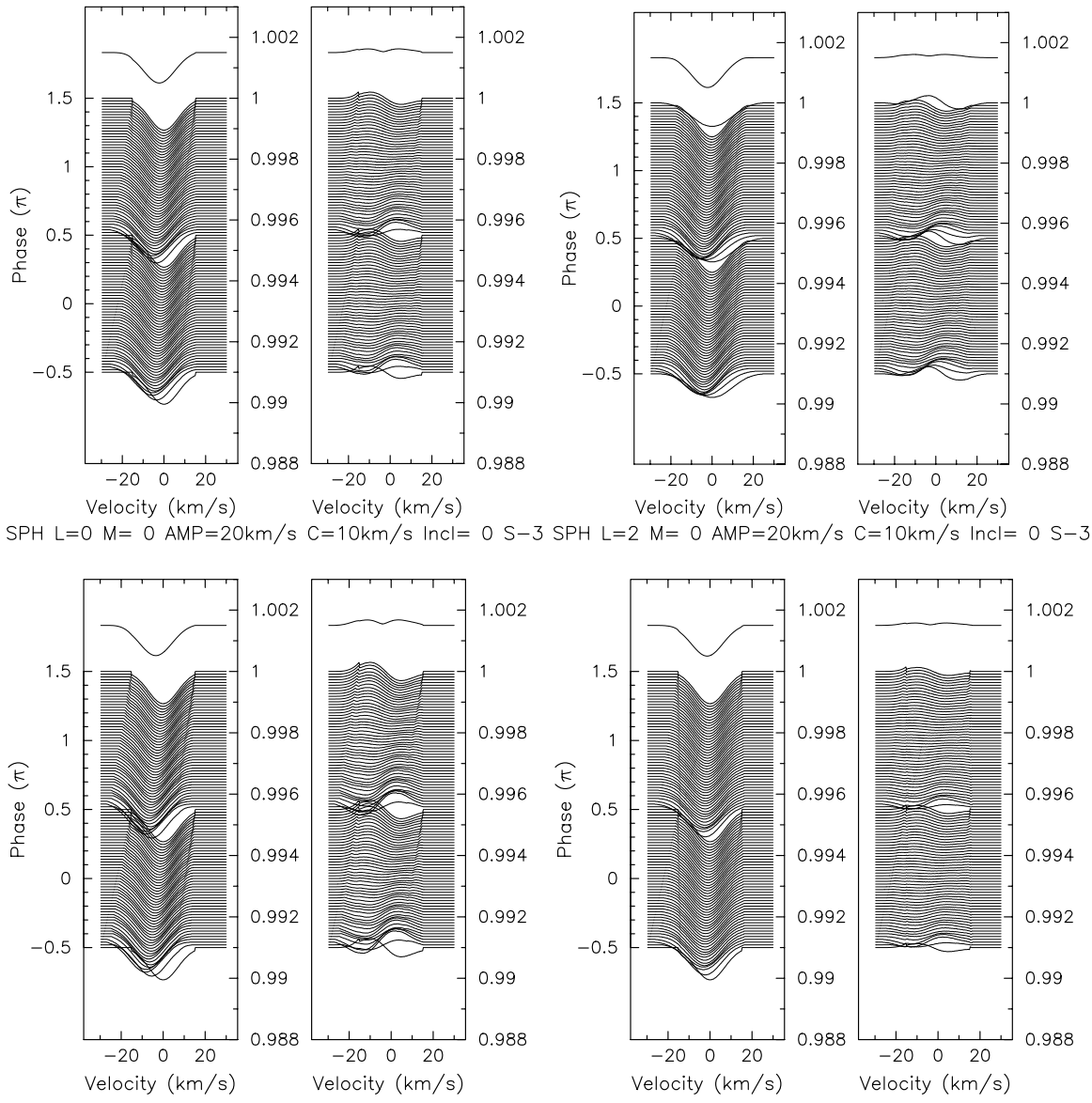

Figure 4. Theoretically expected LPV based on the shock wave hypothesis due to an $\ell=1$, $m=0$ mode (upper left), an $\ell=1, m=1$ mode (upper right), an $\ell=0, m=0$ mode (lower left), and an $\ell=2, m=0$ mode (lower right). The pulsation amplitude is assumed to be $20 \mathrm{~km} \mathrm{~s}^{-1}$ and the line profile is assumed to have an intrinsic line width corresponding to a velocity of $10 \mathrm{~km} \mathrm{~s}^{-1}$. The aspect angle between the pulsation axis and the line-of-sight is assumed to be $30^{\circ}$ for the $\ell=1, m=0$ mode, $90^{\circ}$ for the $\ell=1, m=1$ mode, and $0^{\circ}$ for the $\ell=0, m=0$ mode and the $\ell=2, m=0$ mode. For each mode the LPV for one pulsation cycle is shown on the left panel, and the differences between the LPV and the average profile (shown top left) are displayed in the right panel. Clearly, the LPV move just from blue-to-red. The top of the right panel for each mode shows the standard deviation.

\section{Discussion}

Our new interpretation can be tested observationally by a detailed investigation of LPV of various spectral lines. High spectral resolution, high time resolution, high S/N spectroscopic observations are definitely desired. They will certainly improve our understanding of physics of roAp stars. One example of observational test may be detection of a phase lag between the Nd III and Pr III lines and the other normal lines. Since we are assuming in our model that a shock wave kicks up the Nd III and Pr III line forming layers, it must take a certain time for the shock wave to propagate from its generation layer to the Nd III and Pr III line forming layer. Hence there must be a time lag between the bluest phase of the Doppler shift of the $\mathrm{H} \alpha$ line and that of the Nd III and $\mathrm{Pr}$ III 
lines. If the Nd III and Pr III are formed at $1000 \mathrm{~km}$ above the shock generation layer, the time lag is expected to be of the order of $100 \mathrm{~s}$. This corresponds to a phase lag of $\sim 0.1$. The question is how much mixing the shocks may cause. Further careful consideration is definitely necessary.

\section{References}

Balona, L.A. 2002, MNRAS 337, 1059

Balona, L.A. \& Laney, C.D. 2003, MNRAS 344, 242

Balona, L.A. \& Zima, W. 2002, MNRAS 336, 873

Balona, L.A., James, D.J., Motsoasele, P., Nombexeza, B., Ramnath, A., van Dyk, J. 2002, MNRAS 333, 952

Kochukhov, O. \& Ryabchikova, T. 2001a, A\& $A p$ 374, 615

Kochukhov, O. \& Ryabchikova, T. 2001b, A\&A Ap 377, L22

Kochukhov, O., Landstreet, J.D., Ryabchikova, T., Weiss, W.W., Kupka, F. 2002, MNRAS 337, L1

Kurtz, D.W. 1978, IBVS 1436

Kurtz, D.W. 1982, MNRAS 200, 807

Kurtz, D.W., Elkin, V.G., Mathys, G. 2003 MNRAS 343, L5

Kurtz, D.W., Elkin, V.G., Mathys, G. 2004 MNRAS submitted

Leroy, J.L., Bagnulo, S., Landolfi, M., Landi Degl'Innocenti, E. 1994, A\&̛Ap 284, 174

Mkrtichian, D.E., Hatzes, A.P., Kanaan, A. 2003, MNRAS 345, 781

Sachkov, M., Ryabchikova, T., Kochkokhov, O., Weiss, W.W., Reegan, P., Landstreet, J.D. 2004, in: D.W. Kurtz \& K.R. Pollard (eds.), Variable stars in the local group, ASP Conf. Ser. (San Francisco: Astron. Soc. Pacific), vol. 310, p. 208

Savanov, I.S., Malanushenko, V.P., \& Ryabchikova, T.A. 1999, Aston. Letters 25, 802

Shibahashi, H. 2003, in: L.A. Balona, H.F. Henrichs \& R. Medupe (eds.), Magnetic fields in O, $B$ and $A$ stars: Origin and connection to pulsation, rotation and mass loss, ASP Conf. Ser. (San Francisco: Astron. Soc. Pacific), vol. 305, p. 389

Shibahashi, H., Kurtz, D., Kambe, E., Gough, D. 2004. in: D.W. Kurtz \& K.R. Pollard (eds.), Variable stars in the local group, ASP Conf. Ser. (San Francisco: Astron. Soc. Pacific), vol. 310 , p. 287

Vogt, S.S. \& Penrod, G.D. 1983, ApJ 275, 661 